\title{
Testing of a Prototype Lost $\alpha$-Particle Diagnostic Utilizing an Imaging Bolometer
}

\author{
Evgeny A. DRAPIKO, Byron J. PETERSON, Andrey G. ALEKSEYEV ${ }^{1)}$, Mamiko SASAO ${ }^{2)}$ \\ and Atsushi OKAMOTO ${ }^{2)}$ \\ National Institute for Fusion Science,322-6 Oroshi-cho, Toki 509-5292, Japan \\ 1) TRINITI OFTR, Troitsk, Moscow Region 142190, Russia \\ ${ }^{2)}$ Tohoku University, Sendai 980-8579, Japan
}

(Received 14 December 2009 / Accepted 2 April 2010)

\begin{abstract}
A multi-channel version of the multi foil thermal detector (MFTD) prototype has been developed and tested at the FNL ion accelerator in Tohoku University, which uses an imaging bolometer as a thermal sensor and 2dimensional set of the foils with the different thicknesses as an energy discriminator for alphas. A Phoenix IR camera from FLIR Systems had been used for monitoring the temperature profile variations under the ion beam irradiation. The results obtained showed that the tested foils are applicable for the MFTD design.
\end{abstract}

(c) 2010 The Japan Society of Plasma Science and Nuclear Fusion Research

Keywords: ITER, diagnostic, lost alpha, infrared imaging bolometer

DOI: $10.1585 /$ pfr.5.S2096

\section{Introduction}

A lost alphas diagnostic is one of the high priority tasks for ITER which still has no satisfactory solution. The multi-foil thermal detector proof of principle experiment [1] has resulted in a series of experiments with the multi-channel system utilizing the imaging infrared video bolometer (IRVB) [2]. The foil stack of several foils of different thicknesses acts like an alphas particle energy discriminator. Using the FNL device as the source of alpha particles with predefined energies we have tested a number of foils of different thicknesses and alpha particle energies. Previously shown advantages of high temperature operation regimes of an IRVB [3] were used to increase the sensitivity.

Several successful campaigns of operation of imaging bolometers at LHD and JT-60U devices give the confidence in the applicability of thin metallic foils as parts of a MFTD.

The latest orbit calculations also show a good chance for MFTD to be used in a tokamak device like ITER.

\section{Experiments Description}

Two successful experiments were held at different times in Tohoku University. In the first case we used a rectangular $(6 \times 9 \mathrm{~cm}) 0.6 \mu \mathrm{m} \mathrm{Pt}$ foil as a reradiating one blackened with the graphite and mounted in a copper frame. The reradiating foil was heated up by four special vacuum compatible ceramic heaters up to $220^{\circ} \mathrm{C}$. Stopping foils were mounted in a masked copper frame with $5 \mathrm{~mm}$ holes making a 2-d set of foils with different thicknesses as an energy discriminator. Figure 1 shows the experiment layout

author's e-mail: drapiko.evgeny@lhd.nifs.ac.jp

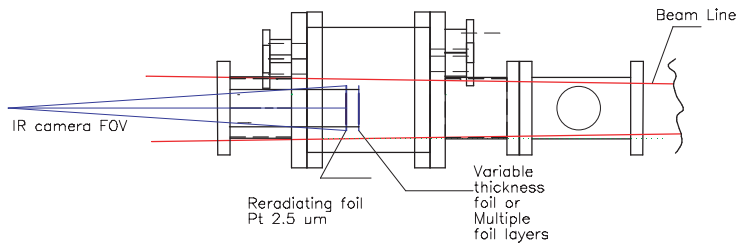

Fig. 1 Experiment layout.

for both cases. In the second experiment we used a reradiating foil masked by the same pattern copper frame as the stopping foils were. That was used to decrease the thermal diffusion from different spots on the foil.

\section{First Experiment}

Figure 2 shows the thicknesses map of the foil stack. Totally 8 different thicknesses, namely $0.8,1.5,2,2.3,2.8$, 3 , 3.8, 4.5, $5.3 \mu \mathrm{m}, 3$ different materials namely $\mathrm{Al}(0.8$, $1.5 \mu \mathrm{m}), \mathrm{Pt}(2 \mu \mathrm{m})$ and $\mathrm{Au}(3 \mu \mathrm{m})$ were used. Energies achieved at that time were $0.8,0.9,1,1.1,1.2,1.3,1.5$, 1.7 and $1.9 \mathrm{MeV}$. Beam current for both experiments was $2 \mu \mathrm{A}$.

A very strong beam profile dependence on the ion energy had been discovered at the FNL which results in quite remarkable difficulties for the quantitative analysis of the data obtained. In order to minimize the effect of beam power and profile variations, the beam was swept by the electric magnets in 2 directions, thus providing relatively uniform irradiation of the foils.

However the qualitative results were quite informative. Figure 3 shows the resulting signal intensity on the reradiating foil irradiated by 0.8 and $1.2 \mathrm{MeV}$ alphas. 


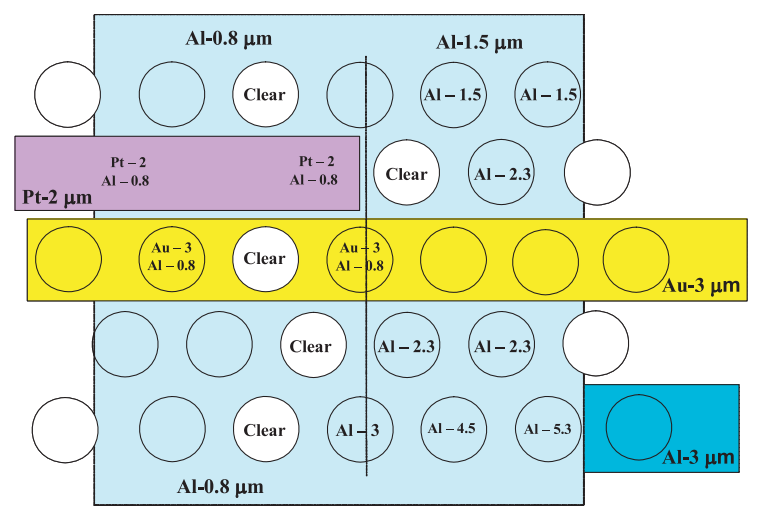

Fig. 2 Discriminating foils thicknesses map.
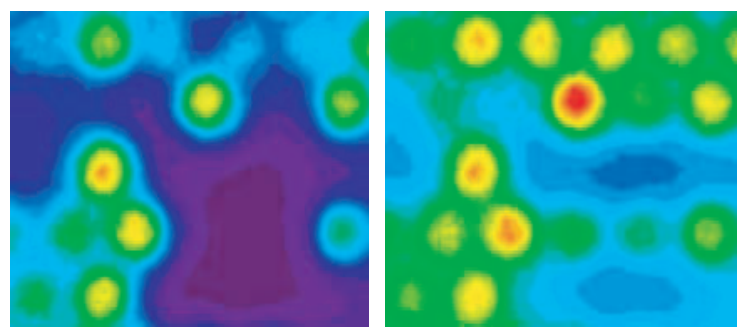

Fig. 3 Signal intensity for 0.8 (left) and 1.2 (right) $\mathrm{MeV}$ alphas.

Comparing with the thicknesses map picture we can state that only clear and thin $(0.8$ and $1.5 \mu \mathrm{m})$ spots are heated in case of $0.8 \mathrm{MeV}$. In the higher energy case $(1.2 \mathrm{MeV})$ some thicker spots like 2 and $2.3 \mu \mathrm{m}$ start to warm up.

On the highest achieved energy of $1.9 \mathrm{MeV}$ almost all the spots are heated except the thickest having $5.3 \mu \mathrm{m}$ of aluminum and $3 \mu \mathrm{m}$ of gold.

Also the thermal diffusion problem on the reradiating foil was found. So, some low signal level spots were completely obscured by the high signal level peaks from clear or thin spots.

\section{Second Experiment}

In order to minimize the thermal diffusion on the reradiating foil it was mounted in between copper masks identical to those holding the discriminating foils and tighten with several little bolts to provide good thermal contact.

In the Fig. 4 is shown the thicknesses map for the discriminating stacks.

In that case we have used 5 different thicknesses ( 0.8 , $1.4,1.5,1.6,2,2.3$ and $2.8 \mu \mathrm{m}$ ) using the same materials as in first experiment. Following alphas energies were used: $0.8,0.9,1.1,1.4,1.9,2.2,2.5 \mathrm{MeV}$. The beam sweeping was not available for that experiment. In order to normalize the profiling effect of the beam it was defocused to become spread and data was acquired line by line horizontally. The reradiating foil was heated up to $200^{\circ} \mathrm{C}$. The signal levels for $0.9 \mathrm{MeV}$ and $1.7 \mathrm{MeV}$ alphas are shown in Fig. 5.

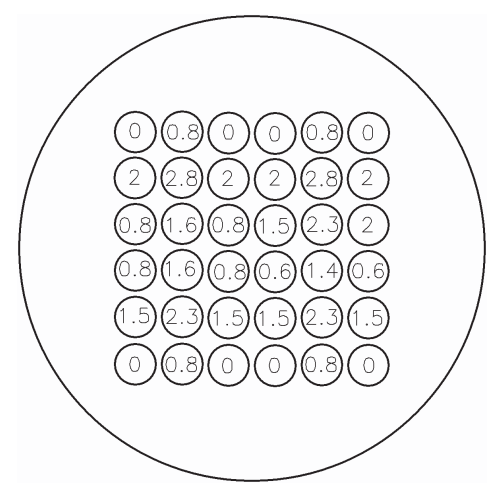

Fig. 4 Discriminating foil thicknesses map.
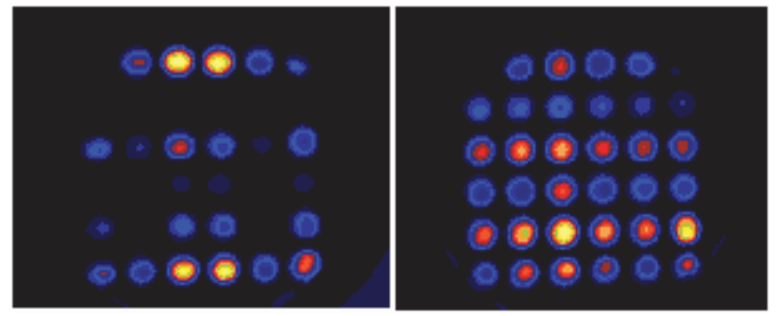

Fig. 5 Signal intensity for 0.9 (left) and 1.7 (right) $\mathrm{MeV}$ alphas.

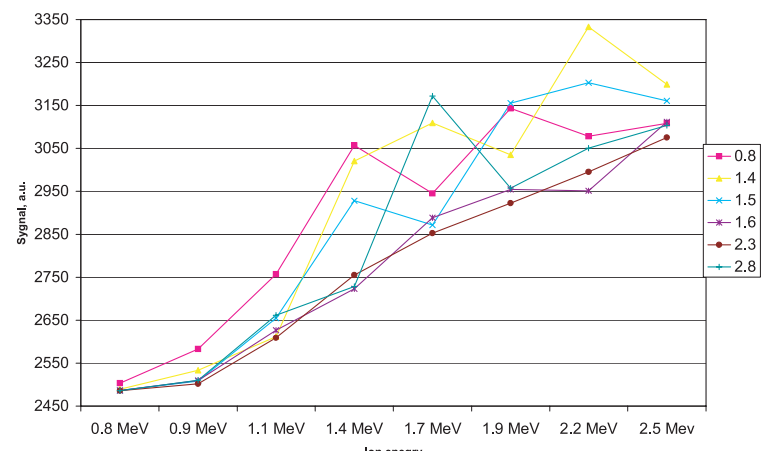

Fig. 6 Signal level over ions energies.

Compared to the thicknesses map we can say that qualitatively the overall picture doesn't change. Clear or thin spots heat up at lower energies and thicker spots heat up at higher energies. The foil thicknesses were decreased to use the energy range of the FNL accelerator more precisely.

Figure 6 shows the signal levels for different thicknesses over the ions energy. Because of the beam sweeping was not possible for that experiment beam profiling effect was very strong and resulted plot has many local peaks. But general trend is absolutely clear.

\section{Conclusion}

The results obtained showed that the tested foils are applicable for the MFTD design. The IR imaging bolometer proved to be an efficient tool for monitoring of energetic 
light ion fluxes. But the absolute accuracy of the beam power measurements was poor due to a number of uncertainties related to the beam power profile and instability, thermal diffusion and the temperature profile of the reradiating foil. The channel separation using the copper mash (second experiment) for the reradiating foil was efficient to decrease the heat diffusion. The beam profile problem is still the issue to be solved in future experiments. The high sensitivity of the technique in addition to the possibility to look at the absorbing side of the foil could provide a good diagnostic tool for lost alphas without stopping foils, using the tokamak magnetic configuration as a discriminator with proper collimation.

[1] A.G. Alekseyev et al., 30th EPS Conf. on Controlled Fusion \& Plasma Phys., July 7-11, 2003, St.Petersburg, Russia, ECA 27A, P1.171 (2003).

[2] B.J. Peterson et al., Rev. Sci. Instrum. 79, 10E301 (2008).

[3] E.A. Drapiko et al., J. Plasma Fusion Res. SERIES 8, 659 (2009). 